Int. J. Dev. Biol. 51: 191-200 (2007)

doi: $10.1387 / \mathrm{ijdb} .062175 \mathrm{~mm}$

Original Article

\title{
Retinoic acid is required for specification of the ventral eye field and for Rathke's pouch in the avian embryo
}

\author{
MALCOLM MADEN* , AIDA BLENTIC, SUSAN REIJNTJES, SOPHIE SEGUIN, EMILY GALE \\ and ANTHONY GRAHAM \\ MRC Centre for Developmental Neurobiology, King's College London, London, UK
}

\begin{abstract}
We have investigated the role of retinoic acid (RA) in eye development using the vitamin A deficient quail model system, which overcomes problems of retinoic acid synthesising enzyme redundancy in the embryo. In the absence of retinoic acid, the ventral optic stalk and ventral retina are missing, whereas the dorsal optic stalk and dorsal retina develop appropriately. Other ocular abnormalities observed were a thinner retina and the lack of differentiation of the lens. In an attempt to explain this, we studied the expression of various dorsally and ventrally expressed genes such as Pax2, Pax6, Tbx6, Vax2, Raldh1 and Raldh3 and noted that they were unchanged in their expression patterns. In contrast, the RA catabolising enzymes Cyp26A1 and Cyp26B1 which are known to be RA-responsive were not expressed at all in the developing eye. At much earlier stages, the expression domain of $S h h$ in the prechordal plate was reduced, as was $N k \times 2.1$ and we suggest a model whereby the eye field is specified according to the concentration of SHH protein that is present. We also describe another organ, Rathke's pouch which fails to develop in the absence of retinoic acid. We attribute this to the down-regulation of Bmp2, Shh and Fgf8 which are known to be involved in the induction of this structure.
\end{abstract}

KEY WORDS: optic stalk, eye development, vitamin A deficiency, quail embryo

\section{Introduction}

The development of the eye has long been known to be dependent upon retinoic acid signalling, since a litter of pigs without eyes was born to a sow kept on a vitamin A deficient diet (Hale 1933). Subsequent vitamin A deficiency studies, mostly using rats, have revealed a range of ocular defects probably depending upon the level of deficiency including microphthalmia, coloboma, abnormal folding of the retina, anterior chamber defects, gaps between the neural and pigmented retina, thickened retrolenticular membrane filling the vitreous cavity, delayed lens differentiation and a denser periocular mesenchyme (Warkany and Schraffenberger 1946; Warkany et al. 1948; Wilson et al. 1953; Dickman et al. 1997;). The reason why the eye is so susceptible to RA deficiency is most likely because it is a site of intense RA synthesis as all the RA synthesising and catabolising enzymes are expressed there. RA can be detected in both the dorsal and ventral regions of the eye (Marsh-Armstrong et al. 1994;McCaffery etal. 1992) and the relative amounts change with developmental age (McCaffery et al. 1993;Mey et al. 1997). The RA-synthesising enzyme Raldh 1 is expressed in the dorsal retina (McCaffery et al. 1991; McCaffery et al. 1992; Godbout et al.,
1996), Raldh3 is expressed in the ventral retina (McCaffery et al. 1999; Grun et al. 2000; Mic et al. 2000; Suzuki et al. 2000) and Raldh2 is expressed in the optic vesicle (Haselbeck et al. 1999), at the ventral edge of the eye field (McCaffery et al. 1999) and in the periocular mesenchyme (Niederreither et al. 1997; Blentic et al., 2003). Cyp26A1 is expressed in an equatorial region of the mouse eye between the dorsal Raldh1 and ventral Raldh3 synthetic domains (Fujii et al. 1997; de Roos et al. 1999; McCaffery et al. 1999; Wagner et al. 2000), a function fulfilled by Cyp26B1 and $C 1$ in the chick embryo (Reijntjes et al. 2003; Reijntjes et al. 2004) thus creating a RA free region and dividing the eye into three zones.

It was expected therefore that these domains of local RA generation would be involved in patterning the developing eye or perhaps the subsequent retinotectal connections in the dorsoventral (DV) axis. However, individual knockouts of the genes coding for these enzymes have failed to generate dramatic phenotypes probably because each enzyme can compensate for the others in this site of multiple enzyme expression. Thus Raldh 1-/- mice have

Abbreviations used in this paper: RA, retinoic acid; RAR, retinoic acid receptor; VAD, vitamin A deficiency.

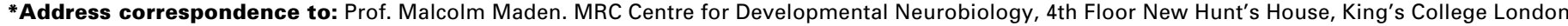
Guy's Campus, London SE1 1UL, UK. Fax: +44-207-848-6798. e-mail: malcolm.maden@kcl.ac.uk
} 
normal eyes and retinotectal connections, unaltered expression of dorsal patterning genes such as Tbx5and, most surprisingly, the early dorsal retina still activated the RARElacZ reporter gene, demonstrating normal levels of RA synthesis (Fan et al. 2003). The latter could be produced by a transient domain of expression of Raldh3in the dorsal retinal pigmented epithelium as RA activity at later stages did disappear in the Raldh1-/- eye. The Raldh3/embryo has a mild shortening of the ventral retina, an abnormal retrolenticular membrane and thickening of the ventral periocular mesenchyme caused by the absence of apoptosis (Dupe et al., 2003; Matt etal.2005). Patterning gene expression domains such as Vax2(ventral) and Tbx5(dorsal) were unaffected. The Raldh2/- embryonic eye had a defect. Here, the optic vesicle failed to invaginate into the optic cup and no lens formed suggesting a role for RA in this early morphogenetic event (Mic et al. 2004). However, again various eye genes were expressed in their normal domains - Pax6, Six $3, R x$ and Mitfand it seems that the presence of normal levels of Raldh 1 and Raldh3in the developing retina compensates for the loss of RA synthesised by Raldh2 (Matt etal., 2005). Rahlh 1/ Raldh3double mutant embryos showed a greater degree of abnormality in the same structures as the single Raldh3 mutant with no additional regions of abnormality and, again, patterning genes such as Tbx5, Vax2and Pax2were normal (Matt et al., 2005; Molokov et al., 2006). This suggests a functional redundancy between these two Raldhs. In the triple mutant there is the first suggestion of a failure of patterned structures with the ventral optic cup failing to invaginate although these embryos had been partially rescued by maternal provision of RA (Molotkov et al., 2006). Here again, the ventral gene Vax2 was still expressed.

Patterning defects as well as general growth failures have also been reported in retinoic acid receptor (RAR) and retinoid $X$ receptor $(R X R)$ null mutant mice. The least affected single mutants were the RAR $\beta$-/- embryos which displayed an abnormal retrolenticular mass of pigmented tissue in the anterior chamber of the eye (Ghyselinck et al. 1997). The most affected single mutants were the RXR $\alpha-/-$ mice which displayed abnormal eyelids, agenesis of the anterior chamber of the eye, coloboma of the optic nerve and shortening of the ventral retina (Kastner et al. 1994). These $R X R$ abnormalities were increased in a graded manner in double mutant embryos. Thus the RAR $\beta / R A R \gamma$ mutants, $\mathrm{RXR} \alpha / \mathrm{RAR} \gamma$ or $\mathrm{RXR} \alpha / \mathrm{RAR} \alpha$ mutants had a very much smaller ventral retina as well as more severe anterior chamber abnormalities (Kastner et al. 1994; Lohnes et al. 1994; Kastner et al. 1997). Therefore from the point of view of patterning, the ventral retina seems to require $R X R \alpha$ and RAR $\gamma$ or RAR $\alpha$ and it is surprising that such a defect had not been seen in vitamin $A$ deficient (VAD) studies as the RAR/RXR knockouts are generally considered to recapitulate all of the VAD phenotypes.

However, a ventral abnormality has been seen after inhibition of RA synthesis with citral, a competitive inhibitor of the aldehyde dehydrogenases. When administered to the zebrafish embryo this compound caused a complete failure of the ventral half of the eye to develop leading to dorsal half eyes (Marsh-Armstrong et al. 1994). Another striking patterning result in zebrafish is the converse, namely a duplicated double eye which developed after systemic treatment of embryos with RA at a precise time during early somitogenesis (Hyatt et al. 1992). These duplicated eyes showed the characteristics of double ventral eyes and local application of RA to the dorsal eye induced an ectopic choroid fissure (Hyatt et al. 1996). Since the usual effect of excess RA administered to other vertebrate embryos is absence of eyes or microphthalmia (Shenfelt 1972; Rosa et al., 1986; Durston et al. 1989; Sive et al. 1990; Sulik et al. 1995; Eagelson et al., 2001) it is important to consider whether these fascinating patterning results on zebrafish are unique to that species or more generally applicable.

To this end we have examined eye development in VAD quail embryos. These embryos are derived from adults fed a VAD diet and contain no detectable retinoids (Dong and Zile 1995; Chen et al. 1996). Since the supply of RA is eliminated, this model system abrogates the problem of redundancy of the RA synthesising enzymes or the RA receptors. We and others have used this model system extensively to characterise the regions of the early embryo which develop abnormally in the absence of RA. These include the cardiovascular system (Zile et al. 2000), the somites (Maden et al. 2000), the hindbrain (Maden et al. 1996; Gale et al. 1999;), the pharyngeal arches (Quinlan et al. 2002) and spinal cord (Wilson et al. 2004). We report the observation that the VAD quail fails to develop the ventral part of the retina including the choroid fissure and that this is likely to be caused by an earlier defect at the ventral midline of the developing central nervous system involving a narrowing of the Shhdomain. This is the first report of such an abnormality in a VAD system and accords with the receptor knockout studies on mouse and the zebrafish inhibition studies described above. We also report that Rathke's pouch, part of the system which produces the pituitary, is also absent in these embryos, highlighting another organ whose development depends upon RA signalling.

\section{Results}

\section{Histological observations}

In unstained, fixed embryos it is possible to see the basic structure of the developing eye (Fig. 1A). The bilayered retina with the ventral choroid fissure (arrowhead, Fig. 1A) gives the eye a tear-drop shape and the lens fills up the internal space. It was readily apparent that the eyes of $\mathrm{VAD}$ embryos were highly abnormal (Fig. 1B). The retina seemed to be much thinner than normal creating a larger ocular space in which the lens was floating and most strikingly there seemed to be a complete absence of the choroid fissure. Thin plastic sections were prepared in order to confirm these impressions. Fig. 1F shows a normal quail stage 20 eye. The neural and pigmented retina are fused together, there is a normal choroid fissure (Fig. 1G) and the eye is surrounded by neural crest derived periocular mesenchyme. In the stage 20 VAD eye the neural retina is not completely fused to the pigmented retina, the lens is not solid but has a cavity in the middle and the whole of the ventral part of the eye is missing including the choroid fissure (Fig. $1 \mathrm{H})$. A close up of the ventral region shows apoptotic cells with condensed spherical nuclei (Fig. 1I, J).

It is possible to rescue these VAD embros with a single injection of retinol prior to the 5-6 somite stage whereupon all the abnormalities in the different organ systems are completely corrected (Gale et al., 1999). This confirms that the abnormalities are due to the lack of retinoids rather than any other secondary alteration. We performed such rescues here with a single injection 
of retinol prior to stage 8 into VAD embryos and in unstained specimens it was clear that the eyes had recovered (Fig. 1C). There was a choroid fissure present (arrowhead, Fig. 1C), the retina was thick and the lens filled the ocular space. We also allowed some embryos to grow up for a considerably longer period of time to ensure that the eyes continued normal development. It is striking that after a single injection of retinol the embryos continued to develop normally, forming perfect embryonic structures including the eyes on day 7 (Fig. 1D, E). Such older embryos were, however, often slightly smaller in overall dimensions than normal.

\section{Gene expression analyses}

Because of this failure of the ventral part of the eye including the optic fissure to form in VAD embryos we examined the expression of genes known to be expressed dorsally and ventrally and potentially involved in patterning of the early eye. In these gene expression analyses 3-5 embryos per stage were used.

We first examined the two retinoic acid synthesising enzymes which are expressed in dorsal and ventral domains, namely Raldh1 and Raldh3. Raldh1 was expressed in the VAD embryo at the normal time, around stage 12 and in its normal dorsal domain (Fig. 2A, B) although the expression extended slightly further ventrally as if the dorsal domain was larger than normal. With regard to the ventrally expressed Raldh3, one might have predicted that it would not appear at all in VAD embryos but this was clearly not the case (Fig. 2C, D). However, in the normal eye there is a mid-ventral gap in expression caused by the developing choroid fissue (Fig. 2C, arrowhead) which is not apparent in the VAD eye where the expression is continuous because there is no choroid fissure (Fig. 2D). The ventral ectodermal expression domain of Raldh3 which will eventually move into the nasal pit and Rathke's pouch is also noticeably smaller in the VAD embryo at this stage (Fig. 2D, arrow). Therefore neither of these enzymes are regulated by the absence of their substrate, but both suggest an alteration of the structure of the eye in the ventral region.

Pax6is a gene crucially involved in the development of the distal retina and whose expression subsequently becomes localised to the dorsal part of the retina. We

Fig. 1. The structure of the stage 20 normal, vitamin Adeficient (VAD) and rescued eye. (A) View of the normal quail eye of an unstained embryo showing the retina (r), lens (I) and choroid fissure (cf). (B) Structure of the VAD eye showing a thin retina ( $r$ ), a smaller lens (I) and a large open space ventrally where the choroid fissure should be. (C) Similar external view of an embryo which was rescued by a single injection of retinol into the egg prior to the 5-6 somite stage. There is a thick retina (r), a lens which fills the optic cavity (I) and the choroid fissure is present as normal (arrowhead). (D) Cartilage stained head of a 7 day rescued embryo showing a completely normal eye after a single injection of retinol at the same early stage as (C). (E) Cartilage stained head of a control 7 day embryo showing that the rescued embryo is normal. (F) $1 \mu \mathrm{m}$ plastic section of a normal quail eye showing the two retinal layers (nr and pr) and the ventral choroid fissure. (G) Close-up of the choroid fissure (cf). (H) $1 \mu \mathrm{m}$ plastic section of a VAD quail eye showing the complete loss of the ventral part of the eye including the choroid fissure, the separation of the two retinal layers ( $\mathrm{nr}$ and pr) and the lens with a large space in the middle. (I,J) Close-up of the cells at the ventral part of the VAD eye showing cells with spherical, dark staining nuclei which are undergoing apoptosis. VAD, vitamin A deficient. examined the expression of this gene in normal and VAD eyes. Pax6 expression begins in the anterior neuroepithelium at stage 8 in both normal and VAD embryos (data not shown). Subsequently when the optic vesicle appears, expression is the same in normal and VAD embryos and remains similar even when the VAD abnormalities have appeared (Fig. 2E, F). Another gene expressed dorsally that we examined was $T b \times 5$. This gene is expressed later than Pax 6 and is confined to the dorsal retina. Again its expression still appeared in the VAD embryo and in the normal dorsal location (Fig. 2G, H).

Two ventrally expressed patterning genes were examined next. The first was Pax2which is involved in the outgrowth of the proximal part of the eye vesicle from the forebrain, that is the optic stalk. It begins to be expressed in both the normal and VAD isthmus at stage 10 (data not shown) and at stage 14 when Pax2 expression is strong in the ventral region of the normal eye it is still expressed in the VAD eye even though the eye cup is clearly thinner and the ventral part is deficient (Fig. 2I, J). At stage 18 when the normal choroid fissure has formed (Fig. $2 \mathrm{~K}$ ) there is still expression in the VAD eye even though there is clearly no fissure (Fig. 2L).

The second ventral gene examined was Vax2 whose expression began in the normal quail eye around stage 14 (Fig. 2M). Expression in the VAD eye did not appear at this stage (Fig. 2N), but by stage 18-20 when normal Vax2 expression was in the
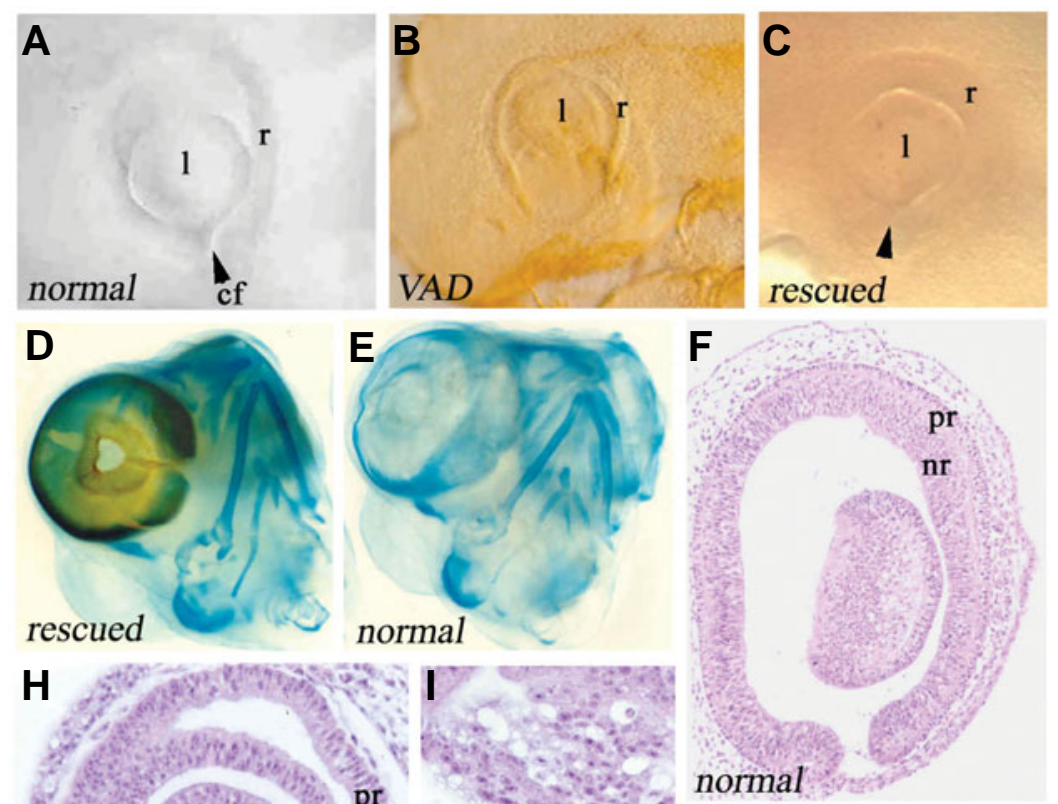

normal

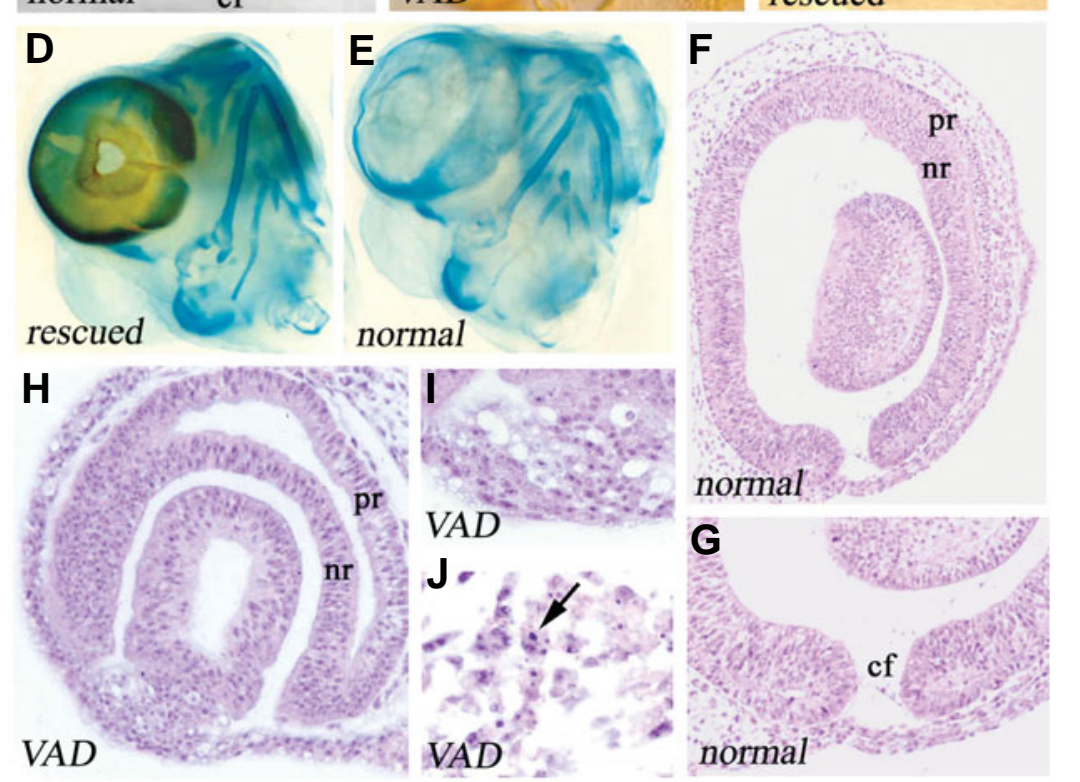


ventral region including the choroid fissure (Fig. 2O) the VAD eye had begun to show some expression (Fig. 2P). Thus the expression of Vax2 was delayed in the VAD eye rather than absent.

Two other enzymes involved in RA metabolism are expressed in the avian eye, Cyp26A1 and Cyp26B1 and their expression was also examined. They have slightly different expression patterns from their equivalent genes in the mouse embryo, but both are induced by RA (Reijntjes et al., 2005). Cyp26A1 is expressed in the dorsal part of the lens and also in a small domain in the ventral part (Fig. 2Q). This expression is absent in the VAD lens (Fig. 2R). Cyp26B1 is expressed in an equatorial region of the avian neural retina (Fig. 2S). This is the domain of expression of Cyp26A1 in the mouse embryo. In the VAD eye Cyp26B1 fails to be expressed at all (Fig. 2T). As they are RA-inducible enzymes, these results imply that both of these domains arise as a result of local RA synthesis

\section{Ventral midline defects}

The lack of any alterations in these patterning genes, apart from the RA-inducible Cyp26genes, suggested that the defect which arose in VAD embryos was realised prior to their appearance. We therefore looked for early anatomical abnormalities and since the eye fields arise from one single midline field under the influence of the prechordal plate (Li et al., 1997) we examined midline structures. Indeed, on closer examination of the ventral aspect of VAD embryos it became clear that they were narrower in the mediolateral dimension as can be seen by comparing Fig. 3A and 3B. These images are of Raldh3 in situs, both taken at the same magnification and show that the overall width of the VAD embryo is less, as well as the width of the posterior projecting domain of Raldh3 expression (bracket in Figs. 3A, B). This could be caused by a loss of tissue and/or decreased signalling at the ventral midline leading to the loss of the ventral part of the evaginating optic primordium. To test this possibility we examined the expression of Shhover these early stages of development in precisely staged pairs of embryos. No difference could be detected in Shh expression between normal and VAD embryos at pre-somite stages or early somite stages. Figs. $3 C$ and $D$ show normal and VAD embryos at the 3-somite stage with indistinguishable Shh expression domains in the midline, but by the 7-somite stage a difference could be detected (Figs $3 E$ \& F) in that the domain was clearly narrower at the anterior end. At the 10-somite stage Figs. $3 \mathrm{G}$ and $\mathrm{H}$ show that this difference is more apparent. By stage 18 Shh expression in the ventral midline of the dien-

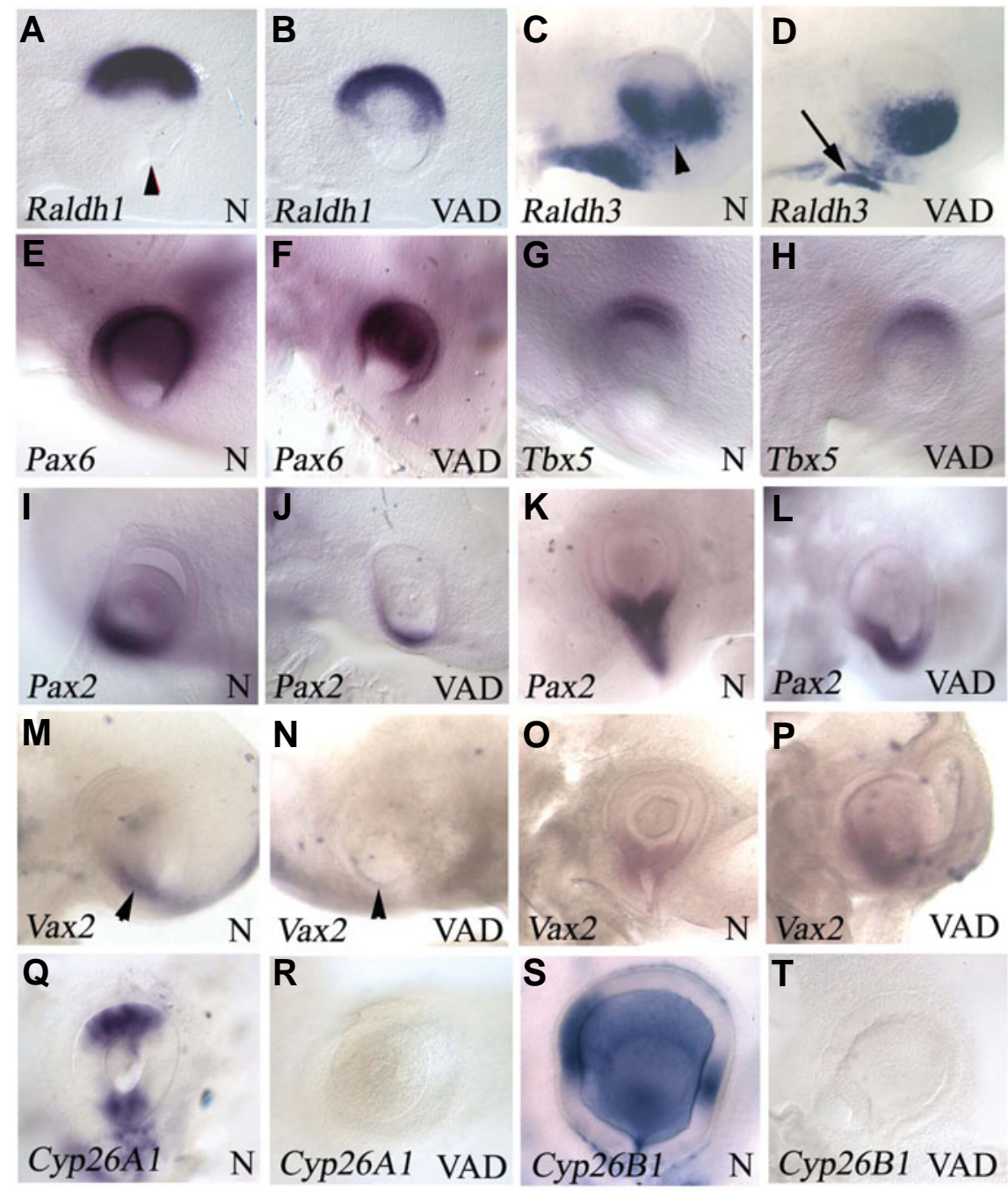

Fig. 2. Gene expression analysis of normal and VAD quail embryos. (A) Normal stage 18 eye showing dorsally restricted Raldh1 expression. The arrowhead shows the choroid fissure. (B) Stage 18 VAD embryo showing a more extensive dorsal expression of Raldh1, a thinner retina and absence of choroid fissure. (C) Normal stage 14 frontonasal region showing expression of Raldh3 in the ventral part of the eye and in the epithelium of the nasal placode and oral region. At the ventral pole of the eve is a gap in Raldh3 expression where the choroid fissure is present (arrowhead). (D) Stage 14 VAD embryo showing Raldh3 expression in the ventral part of the eye, but with no gap between the two parts, that is, no choroid fissure. The ventral epithelial expression is also less extensive (arrow). (E) Pax6 expression in a stage 14 normal embryo in the dorsal region of the eye. (F) Pax6 expression in the dorsal region of a VAD eye. (G) Tbx5 expression in the dorsal region of a normal stage 14 eye. (H) Tbx5 expression in a VAD stage 14 eye. (I) Pax2 expression in the ventral region of a stage 14 normal eye. (J) Pax2 expression in a stage 14 VAD eye, in a smaller ventral domain than normal partly because the retina is thinner and partly because the choroid fissure will not develop. (K) Pax2 expression in the ventral region and choroid fissure of a stage 18 normal eye. (L) Pax2 expression in the ventral region of a stage 18 VAD eye showing the absence of a choroid fissure. (M) Vax2 expression in the ventral region of a stage 14 normal eye. (N) Absence of $\mathrm{Vax} 2$ expression in a stage 14 VAD eye. (0) Vax2 expression in the ventral retina of a normal stage 20 eye. (P) Vax2 expression in the ventral region of a stage 20 VAD eye. (Q) Cyp26A1 expression in a dorsal and ventral region of the stage 18 normal lens. (R) Absence of Cyp26A1 expression in a stage 18 VAD lens. (S) Cyp26B1 expression in the equatorial region of the neural retina of a stage 20 normal eye. (T) Absence of Cyp26B1 expression in a stage 20 VAD eye. Abbreviations: $N$, normal; $V A D$, vitamin A deficient. 
cephalon and midbrain had virtually disappeared (Figs. 3I \& J, arrowheads in $3 \mathrm{~J}$ ) although not in the ventral midline in the hindbrain region (arrow in Fig $3 \mathrm{~J}$ ). This confirms that RA controls gene expression locally rather than globally. Another ventral midline gene, $N k \times 2$. 1 was also virtually eliminated in the anterior head region by stage 18 (Fig. $3 \mathrm{~K}-\mathrm{L}$, arrowheads in $3 \mathrm{~L}$ ), but not more posteriorly in the hindbrain (arrow in Fig. 3L).

\section{Another abnormal region - Rathke's pouch}

This missing region of the ventral eye corresponded precisely to the domain of Raldh3 expression (Fig. 4A). It is possible, therefore, that Raldh3 expression might serve as a marker for regions of the embryo which are also abnormal in the absence of RA. In the head region there are three regions in addition to the ventral eye which express Raldh3, namely the isthmus, the otic vesicle and Rathke's pouch (Grun et al., 2000) (Fig. 4B). The otic vesicle is certainly abnormal and fails to invaginate (Maden et al., 1996; Kil et al. 2005) supporting the idea that Raldh3 serves as a marker of abnormality. On closer examination of the isthmus we could detect no obvious differences between normal and VAD embryos, although a full gene expression and anatomical analysis was not performed. It remains possible that there may be differences to be found in the future and the Raldh3knockout mouse would be a valuable resource for looking at this.

With regard to Rathke's pouch this structure was clearly dependent on RA provided by Raldh3 because it was entirely absent in VAD embryos. Fig. $4 \mathrm{C}$ shows a sagittal section through a normal stage 20 embryo in which the expression of Raldh3 is precisely in Rathke's pouch. Fig. 4D shows a histological section of a normal embryo revealing the in-pushing of the oral epithelium (arrow in Fig. 4D) which creates Rathke's pouch by coming into contact with the thickened ventral forebrain neural epithelium (arrowhead in Fig. 4D). In contrast, a similar section through a VAD embryo (Fig. 4E) shows a thinner forebrain neuroepithelium with no sign of a thickening and an oral epithelium which shows no sign of an in-pushing. This suggests that the early development of the pituitary in terms of these cell interactions and morphogenetic movements is dependent upon RA synthesised by Raldh3.

$B m p 2$ and $F g f 8$ are two genes which are involved in the neuroepithelium/oral epithelium interaction to instigate early pituitary gland development (reviews, Dasen \& Rosenfeld, 1999; Sheng \& Westphal, 1999) so we examined their expression in normal and VAD quail embryos. The wholemount in Fig. 4F shows the normal expression of Bmp2 in Rathke's pouch (arrows in Fig. 4F) and the rest of the head region including the $1^{\text {st }}$ branchial arch (arrowhead in Fig 4F). The absence of Bmp2 expression in the region of Rathke's pouch in the VAD embryo is revealed in Fig. 4G (arrows), but its continued expression in the 1st branchial arch (arrowheads in Fig. 4F \& G). Again, this demonstrates local not global control of gene expression by RA. Fgf8 expression is present in the normal oral epithelium (Fig. $4 \mathrm{H}$, arrow) including the epithelium of the first branchial cleft. This expression is down-regulated in the VAD embryo leaving a small patch of Fgf8 on the external epithelium of the cleft (Fig. 4I, arrow), but other regions such as the isthmus are not affected. As we observed earlier (Fig. 3I, J) Shhexpression, which is another gene involved in pituitary development by
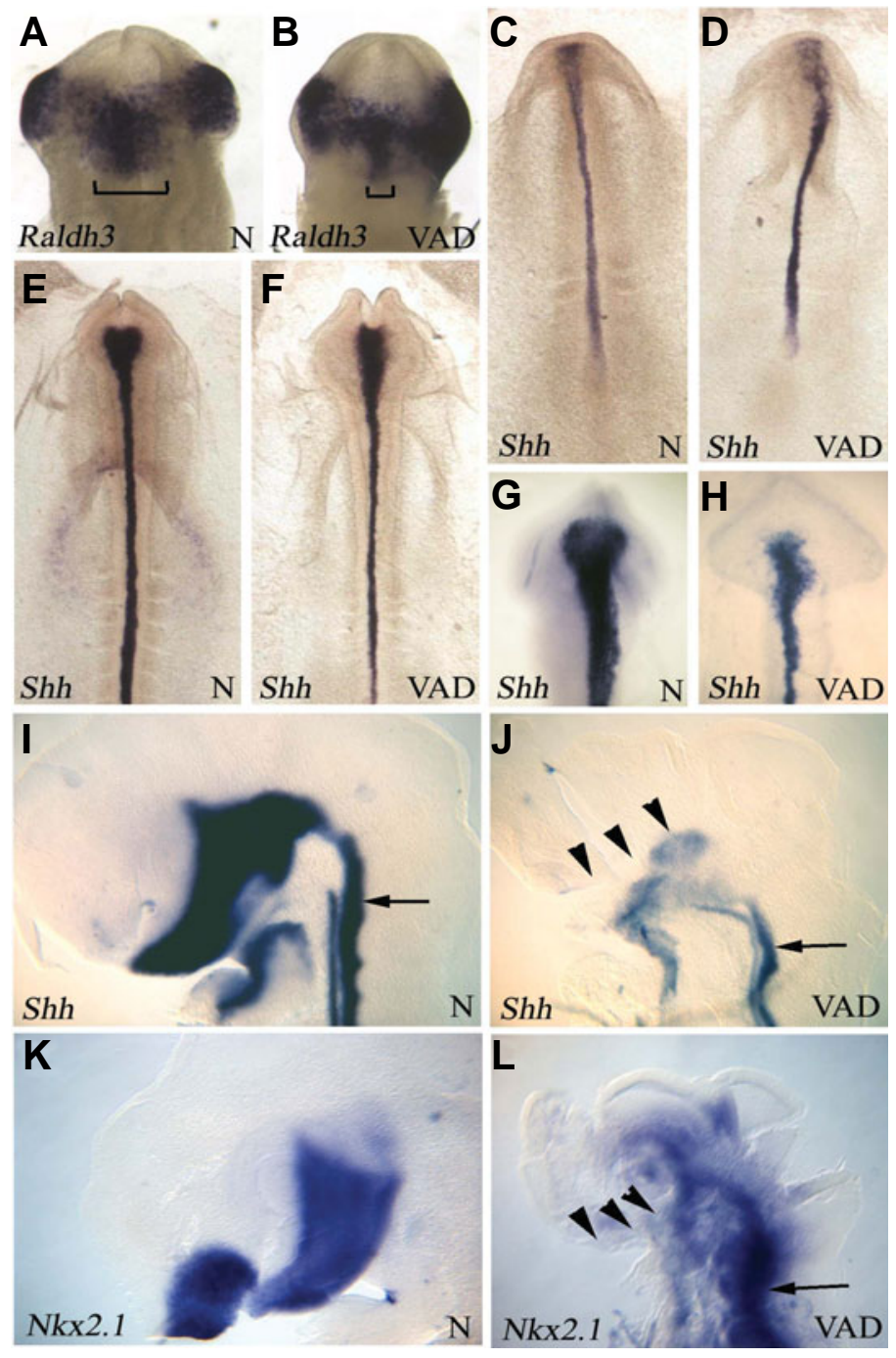

Fig. 3. Gene expression analysis of normal and vitamin A-deficient (VAD) quail embryos. (A) Ventral view of Raldh3 expression in a normal stage 10 embryo showing the width of the extent of expression in the ventral epithelium beneath the developing forebrain. The bracket marks the width of the central domain. (B) Ventral view of Raldh3 expression in a stage 10 VAD embryo at the same magnification as $(A)$, showing how much narrower the extent of expression is and the width of the embryo. The bracket marks the same central domain and is also narrower. (C) Shh expression in a normal 3-somite stage embryo. (D) Shh expression in a 3-somite stage VAD embryo showing little difference from normal. (E) Shh expression in a 7-somite stage normal embryo. (F) Shh expression in a 7-somite stage VAD embryo showing that at the anterior end the Shh expression domain is narrower. (G) Shh expression at the anterior end of a 10-somite stage normal embryo. (H) Shh expression at the anterior end of a 10-somite stage VAD embryo showing a far narrower domain of expression. (I) Lateral view of Shh expression in the anterior region of a stage 18 normal embryo showing expression in the ventral midline of the diencephalon, midbrain and hindbrain (latter marked by an arrow). (J) Strong down-regulation of Shh expression in the diencephalon (arrowheads) and midbrain ventral midline of a stage 18 VAD embryo, but continued expression in the hindbrain (arrow). (K) Lateral view of Nkx2.1 expression in the ventral midline of the diencephalon of a stage 18 normal embryo. (L) Strong down-regulation of Nkx2.1 expression in a stage 18 $V A D$ embryo in the diencephalon (arrowheads), but not in the hindbrain (arrow). Abbreviations as in Fig. 2. 


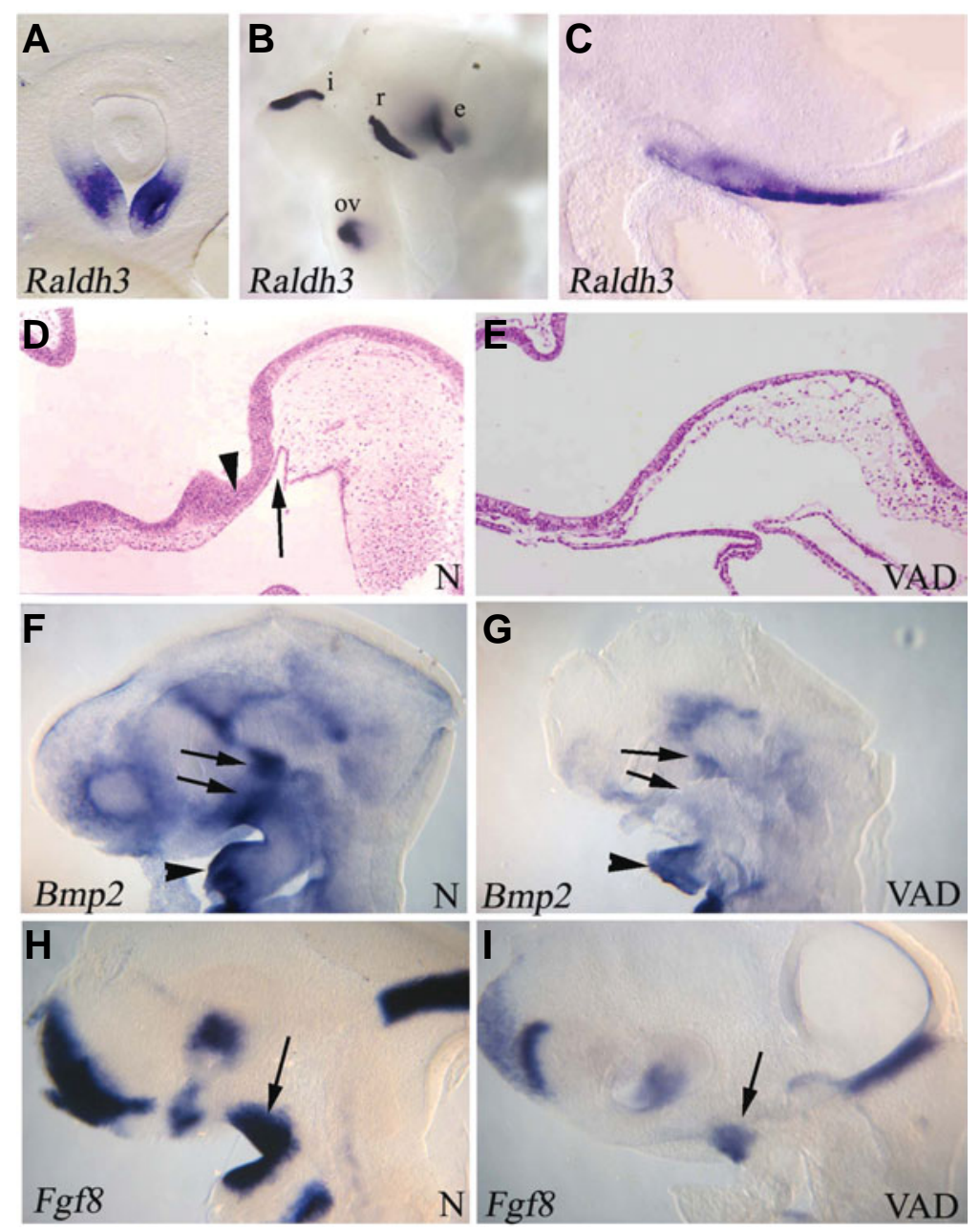

being expressed in the oral epithelium, is also strongly downregulated.

\section{Discussion}

Redundancy among the RA synthesising enzymes, the RALDHs, has made it very difficult to pursue studies on the function of RA in the developing eye as all three Raldhs are expressed there. The absence of one enzyme is compensated for by the presence of at least two others (Sakai et al. 2004; Matt et al., 2005). This has led to the conclusion that RA is not involved in DV specification of the eye despite the observations in the zebrafish embryo that in the presence of excess RA a double ventral eye forms with an expanded optic stalk (Hyatt et al., 1996) and in the presence of citral the ventral half of the eye fails to form (Marsh-Armstrong et al., 1994). To overcome these redundancy problems we have examined eye development in the VAD quail embryo which develops in the absence of RA, by removing the source of RA, namely retinol, from the diet. Under these circumstances none of the RALDHs can function due to the absence of substrate, a situation equivalent to a multiple gene knockout. As a result we find that the ventral half of the eye including the choroid fissure fails to develop and some of the cells in that region undergo apoptosis, presumably those that have failed to be specified appropriately. The remaining portion of the neural and pigmented
Fig. 4. Rathke's pouch and gene expression analysis of normal and VAD quail embryos. (A) Raldh3 expression in the ventral region of a normal stage 18 eye. (B) Lateral view of Raldh3 expression in the head of a stage 18 normal embryo showing the sites of expression in the head, namely the isthmus (i), the dorsal otic vescle (ov), Rathke's pouch (r) and the ventral eye (e). (C) Section through a stage 18 normal embryo showing Raldh3 expression in the oral epithelium of Rathke's pouch. (D) $1 \mu \mathrm{m}$ sagittal plastic section through a stage 18 normal embryo showing the in-pushing of the oral epithelium which creates Rathke's pouch (arrow) and the thickening of the ventral forebrain neuroepithelium (arrowhead) which together generate the pituitary gland. (E) Sagittal section through a stage 18 VAD embryo showing no sign of an in-pushing of the oral epithelium resulting in the absence of Rathke's pouch. (F) Lateral view of the head of a stage 20 normal embryo showing Bmp2 expression in Rathke's pouch (arrows) and 1st branchial arch (arrowhead). (G) Lateral view of the head of a stage 20 VAD embryo showing the downregulation of $\mathrm{Bmp} 2$ expression in the anterior head region and particularly Rathke's pouch (arrows), but not in the $1^{\text {st }}$ branchial arch (arrowhead). (H) Lateral view of a stage 20 normal embryo showing Fgf8 expression in the anterior region, the eye, Rathke's pouch (arrow) and the first branchial arch. (I) Down-regulation of Fgf8 expression in the anterior head of a stage 20 VAD embryo, particularly in the oral region (arrow).

retina are much thinner than normal and there is no real fusion between these two layers. The optic space appears larger and the eye looks empty. The lens still forms although it is more vacuolated rather than being densely packed with cells and perhaps crystallin synthesis, which is known to be regulated by RA (Tini et al. 1993), is downregulated. We conclude therefore that $\mathrm{RA}$ is required for the specification of the ventral retina, the ventral optic stalk and the choroid fissure, the proliferation and growth of the retina and the differentiation of the lens.

It is surprising that such a major defect in the eye structure such as a missing ventral half of the retina and ventral optic stalk has not been seen before in VAD studies in mammals. Only microphthalmia, amongst other defects, has been reported (Warkany and Schraffenberger 1946; Warkany et al. 1948; Wilson et al. 1953; Dickman et al. 1997). It could well be that the histological structure of such eyes has not been studied and that they were, in fact, missing ventral tissue although a general growth inhibition caused by reduced RA signalling is also a possibility (Sen et al. 2005). It is also possible that they were not completely VAD but only partially so and a general growth inhibition to produce microphthalmic eyes occurs at low RA levels, but for ventral defects the absence of RA is required. Curiously, microphthalmia is the classical response of the eye to excess RA signalling caused by altered specification of the neural plate and most clearly seen in Xenopus (Durston et al., 1989; Manns and Fritzsch 1991; Eagelson et al., 2001), caused by a down-regulation of Pax6 and a forebrain marker Xbf1 (Eagleson et al., 2001). But in neither of these situations have patterning aspects of microphthalmic eyes been fully studied so it is not possible yet to determine whether excess RA and a deficiency of RA generate the same precise patterning alterations.

The failure of proliferation of the neuroepithelium is not unique to the retina, but has been observed throughout the CNS (Wilson et al. 2003). After stage 14 the VAD neural tube fails to increase 
its proliferation rate as normal embryo do and as a result the whole neuroepithelium remains a thin tissue. The effect on the lens, however, is specific to the eye because RA is involved in lens development not only because of its ability to regulate crystallin synthesis (Tini et al., 1993), but also because the optic cup and lens is a site of intense RA signalling activity (Enwright and Grainger 2000). In this region, RA signalling is down-regulated in the small eye (Pax6) mutant so RA seems to be downstream of Pax6which explains why Pax6expression seemed unaffected in the VAD embryo. Indeed, neither Raldh 1 nor Raldh3appear in the Pax6 rat mutant (Suzuki et al. 2000). A more dramatic effect of lack of RA signalling on the lens has been seen in mouse embryos where antisense oligonucleotides to retinol binding proteins were injected into the yolk sac. In this case there was a complete failure of lens induction (Bavik et al. 1996).

We examined several other genes thought to be involved in DV specification of the eye, such as Pax2, Tbx5and Vax2and found them also to be expressed in their normal domains, although Vax2 was delayed in its appearance. Tbx5 and Vax2 begin to be expressed quite late in development (stage 14) and so these are unlikely to be involved in eye field specification. Neither was there any change in the expression of these two genes in experiments where RA signalling was reduced using a dnRAR $\alpha$ construct (Sen et al., 2005). On the contrary, Vax2 regulated the expression of the RA synthesising enzymes $R a / d h 1$ and $R a / d h 3$ which is why we saw no change in these two gene expression domains. There was obviously no effect of lack of substrate on the expression of these two enzymes. On the other hand, the two RA catabolising enzymes Cyp26A1 and Cyp26B1 were not expressed so their expression does depend on substrate availability which might be expected since these enzymes are induced by RA (Reijntjes et al. 2005).

Figure 5 depicts a summary diagram of the normal eye (Fig. $5 \mathrm{~A}$ ) and the parts that are missing in the VAD eye (Fig. 5B) namely the ventral optic stalk and ventral retina. This is clearly a patterning defect rather than a proliferation defect and it is interesting to hypothesise how this may have come about. The early eye field is specified as a single unit within the neural plate which splits under the influence of the prechordal plate to emerge as two separate fields ( $\mathrm{Li}$ et al., 1997). Within this emerging eye field RA may be responsible for specifying the ventral optic stalk and ventral retina domains. Pax2 is expressed in the optic stalk (Macdonald et al., 1995) and the failure of this gene to be expressed could be responsible, but $P a x 2$ is expressed in these VAD quails and they are not cyclopic. It is more likely that there is some graded explanation for this phenotype rather than an all-ornone phenomenon and $S h h$ is a likely candidate as it controls DV polarity of the eye in a concentration dependent fashion (Lupo et al. 2005).

Shh is expressed in the midline prechordal plate and induces Pax2 and optic vesicle outgrowth. In the absence of Shh the splitting of the optic vesicles from the single eye field does not take place and cyclopia results (Chiang et al., 1996). Nevertheless distal retinal tissue forms, albeit in the wrong location because of the failure of optic stalk tissue to be induced. Conversely overexpression of Shh causes an expansion of the Pax2domain and other ventral markers including Raldh3, resulting in expanded optic stalks and small eyes due to overproduction of ventral tissue (Ekker et al., 1995; Macdonald et al., 1995; Lupo et al., 2005) and

in the chick embryo the ventral retina is replaced by the optic stalk (Zhang \& Yang, 2001). Shh signalling acts in a graded way to induce these optic fates: at the highest $S h$ levels ventral optic stalk is induced, at lower levels ventral retina is induced and the dorsal retina is unaffected by loss of Shh as is Pax6 expression (Chow \& Lang, 2001; Lupo et al., 2005; Fig. 5C). In vivo there is a high medial to low lateral gradient of Ptc mRNA, the $\mathrm{SHH}$ receptor, along the optic stalk (Zhang \& Yang, 2001). Thus to explain these eye defects we reasoned that $S h h$ levels may be altered in the VAD quails, but certainly not obliterated as the embryos were not cyclopic. This is indeed what was observed. Shh expression was at similar levels, but in a narrower band of prechordal plate mesoderm which would presumably result in overall lower levels of SHH protein concentrations (Fig. $3 \mathrm{E}$ vs $\mathrm{F}$ and $\mathrm{G}$ vs $\mathrm{H}$ ). This would lead to the loss of ventral optic stalk, ventral retina, the preservation of the dorsal retina (Fig. 5C), all of which would occur, not in the absence of Pax2, but at lower levels. If we hypothesise that specification of the dorsal optic stalk takes place at $\mathrm{SHH}$ levels intermediate between ventral retina and dorsal retina then the VAD phenotype becomes explicable, based on lowered SHH levels (Fig. $5 \mathrm{C}$ ). In fact the VAD quails are remarkably similar to the cyclops zebrafish phenotype which is characterised by reduced Shhexpression, reduced Pax2expression and reduced optic stalks (Macdonald et al., 1995).

In eye development RA thus plays a continuing sequence of roles. The first is an early role, at gastrulation stages, perhaps to

\section{A}

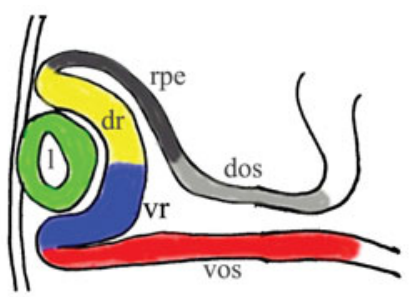

B

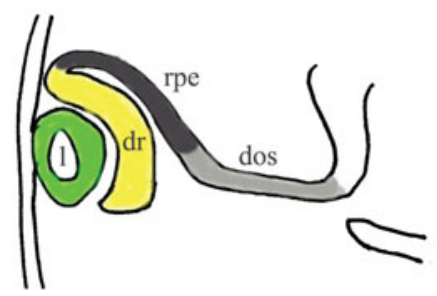

Fig. 5. Drawing of the eye structure in normal and VAD quail embryos and a possible explanation of its formation. (A) Drawing of the different regions within a developing eye. I, lens; rpe, retinal pigment epithelium; dr, dorsal retina; dos, dorsal optic stalk; vr, ventral retina; vos, ventral optic stalk. (B) Drawing of the eye which develops in VAD embryos. The ventral retina and ventral optic stalk are missing. (C) Hypothesis to explain the VAD eye phenotype based on the levels of SHH protein (red gradient). In the normal embryo the dorsal retina (DR) develops irrespective of SHH levels, the dorsal optic stalk (DOS) develops at low levels of SHH, the ventral retina (VR) at intermediate levels and the ventral optic stalk (VOS) at the highest levels. Right drawing: under VAD conditions the levels of SHH are much lower than normal and only the dorsal optic stalk is specified. 
regulate the amount of mesoderm which enters the prechordal plate. Fewer prechordal plate cells will produce a smaller total amount of $\mathrm{SHH}$ protein resulting in the failure of the ventral optic stalk specification or outgrowth. Alternatively there could be reduced levels of prechordal plate signalling in the absence of RA. Indeed, a RA synthesising enzyme, Raldh3 is expressed during gastrulation in Hensen's node (Maden 2004) and in the dorsal lip of the blastopore (Lupo et al., 2005) and early prechordal plate abnormalities in size and signalling properties have been reported in VAD quails (Halilagic et al. 2003). A later role for RA, in addition to retinal growth control, is one we have not approached here and concerns DV pattering of the retina and the retinotopic map through the regulation of the expression of EphB2, EphB3 and ephrinB2 (Sen et al., 2005). Here again, the absence of altered retinotopic maps in Raldh mutant mice may be due to redundancy.

A further defect in the craniofacial region that we observed in these VAD embryos was the failure of Rathke's pouch to develop. The oral epithelium failed to invaginate and approach the ventral forebrain neuroepithelium such that the normally close interaction which results in the development of the pituitary gland could not occur. In contrast to the eye patterning defects, this abnormality has been seen in single Raldh mutants, in this case the Raldh2-/-embryo (Ribes etal. 2006). In the region of Rathke's pouch we observed a loss of Shh, Nkx2.1, Bmp2and Fgf8expression. Each of these genes is known to play a role in the early development of the pituitary gland. Shh expression creates a boundary between the oral epithelium and Rathke's pouch epithelium which acts as an organising centre for further gene induction as well as being expressed in the ventral diencephalon (Treier et al., 1998: 2001) and this was strongly down-regulated in the VAD embryo. Bmp2 is expressed in the epithelium of Rathke's pouch itself (Treier et al., 1998: 2001) and this was down-regulated. Nkx2. 1 expression in the ventral diencephalon is required for Fgf8 maintenance in the same region (Ericson et al., 1998; Takuma et al., 1998) and both of these were also down-regulated. Thus many of the known key players in the early steps of pituitary gland development are under the control of RA. Again, as has been occasionally seen in eye development, both an excess and a lack of RA can cause the same phenotype and in the case of the pituitary excess RA has also been reported to cause an inhibition of its development (Eagelson et al., 2001).

In conclusion we have shown through the use of the VAD quail embryo that RA signalling is required for the development of the ventral region of the eye and Rathke's pouch. This model in which the ligand, RA, is absent provides a simple system with which to overcome the problem of RA synthesising enzyme redundancy, a problem which may hide regions of the embryo requiring $\mathrm{RA}$ signalling.

\section{Materials \& Methods}

\section{Embryos}

Fertilised hen's eggs and quail's eggs were obtained from a local farm supplier. VAD quail eggs were obtained from a colony kept at King's College London. The adults in this colony were fed a diet devoid of any vitamin A except for the addition of $10 \mathrm{mg} / \mathrm{kg}$ all-trans-retinoic acid to the diet. All embryos were incubated at $37^{\circ} \mathrm{C}$ and staged according to Hamburger and Hamilton (Hamburger and Hamilton 1951).

\section{In situ hybridisation}

After fixation of the embryos in 4\% paraformaldehyde, wholemount in situ hybridisation was carried out according to standard protocols using digoxigenin labelled probes from plasmids available in the laboratory. The Vax2 and Tbx5probes were kindly provided by Dr C. Cepko. After in situ hybridisation embryos were placed into $80 \%$ glycerol and were either studied and photographed whole, after mid-sagittal sectioning or after vibratome sectioning at $80 \mu \mathrm{m}$. For gene expression analyses 3-5 embryos per stage per gene were used.

\section{Histology}

Embryos were sectioned at $1 \mu \mathrm{m}$ in plastic using a Technovit 8100 resin kit (TAAB laboratories).

\section{Acknowledgements \\ We thank Dr C. Cepko for the Vax2 and Tbx5 plasmids.}

\section{References}

BAVIK C, WARD SJ and CHAMBON P. 1996. Developmental abnormalities in cultured mouse embryos deprived of retinoic acid by inhibition of yolk-sac retinol binding protein synthesis. Proc Nat/ Acad Sci USA 93:3110-3114.

BLENTIC A, GALE E and MADEN M. 2003. Retinoic acid signalling in the avian embryo identified by sites of expression of synthesising and catabolising enzymes. Dev Dynam 227:114-127.

CHEN Y-P, DONG D, KOSTETSKII I and ZILE MH. 1996. Hensen's node from vitamin A-deficient quail embryo induces chick limb bud duplication and retains its normal asymmetric expression of sonic hedgehog (Shh). Dev Bio/173:256264.

CHIANG C, LITINGTUNG Y, LEE E, YOUNG KE, CORDER JL, WESTPHAL H and BEACHY PA. 1996. Cyclopia and defective axial patterning in mice lacking Sonic hedgehog gene function. Nature 383:407-413.

CHOW RL and LANG RA. 2001. Early eye development in vertebrates. Ann Rev Cell Dev Biol 17:255-296.

DE ROOS K, SONNEVELD E, COMPAAN B, TEN BERGE D, DURSTON AJ and VAN DER SAAG PT. 1999. Expression of retinoic acid 4-hydroxylase (CYP26) during mouse and Xenopus laevis embryogenesis. Mech Dev82:205-211.

DASEN, JS and ROSENFELD, MG. 1999. Combinatorial codes in signalling and synergy: lessons from pituitary development. Curr Opinion Gen. Dev 9:566574

DICKMAN ED, THALLER C and SMITH SM. 1997. Temporally-regulated retinoic acid depletion produces specific neural crest, ocular and nervous system defects. Development 124:3111-3121.

DONG D and ZILE MH. 1995. Endogenous retinoids in the early avian embryo. Biochem Biophys Res Comm 217:1026-1031.

DUPE V, MATT N, GARNIER J-M, CHAMBON P, MARK $M$ and GHYSELINCK NB. 2003. A newborn lethal defect due to inactivation of retinaldehyde dehtdrogenase type 3 is prevented by maternal retinoic acid treatment. Proc Nat/Acad SciUSA 100:14036-14041.

DURSTON AJ, TIMMERMANS JPM, HAGE WJ, HENDRICKS HFJ, DE VRIES NJ, HEIDEVELD M and NIEUWKOOP PD. 1989. Retinoic acid causes an anteroposterior transformation in the developing nervous system. Nature 340:140 144

EAGELSON GW, JOHNSON-MEETER LJ and FRIDERES J. 2001. Effects of retinoic acid upon eye field morphogenesis and differentiation. Dev Dynam221, 350-364.

EKKER SC, UNGAR AR, GREENSTEIN P, VON KESSLER DP, PORTER JA, MOON RT and BEACHY PA. 1995. Patterning activities of vertebrate hedgehog proteins in the developing eye and brain. Curr Bio/5:944-955.

ENWRIGHT JF and GRAINGER RM. 2000. Altered retinoid signaling in the heads of small eye mouse embryos. Dev Bio/221:10-22.

ERICSON J, NORLIN S, JESSELL TM and EDLUND T. 1998. Integrated FGF and BMP signalling controls the progression of progenitor cell differentiation and the emergence of pattern in the embryonic anterior pituitary. Development 125:1005-1015 
FAN X, MOLOTKOV A, MANABE S, DONMOYER CM, DELTOUR L, FOGLIO MH, CUENCA AE, BLANER WS, LIPTON SA and DUESTER G. 2003. Targeted disruption of Aldh1a1 (Raldh1) provides evidence for a complex mechanism of retinoic acid synthesis in the developing retina. Mol Cel/ Bio/23:4637-4648.

FUJII H, SATO T, KANEKO S, GOTOH O, FUJII-KURIYAMA Y, OSAWA K, KATO $S$ and HAMADA H. 1997. Metabolic inactivation of retinoic acid by a novel $P 450$ differentially expressed in developing mouse embryos. EMBO J16:4163-4173.

GALE E, ZILE M and MADEN M. 1999. Hindbrain respecification in the retinoiddeficient quail. Mech Dev89:43-54.

GHYSELINCK NB, DUPE V, DIERICH A, MASSADDEQ N, GARNIER J-M, ROCHETTE-EGLY C, CHAMBON P and MARK M. 1997. Role of the retinoic acid receptor beta (RARb) during mouse development. Int J Dev Bio/41:425447.

GODBOUT R, PACKER M, POPPEMA S and DABBATH L. 1996. Localization of cytosolic aldehyde dehydrogenase in the developing chick retina: in situ hydridisation and immunohistochemical analyses. Dev Dynam 205:319-331.

GRUN, F., HIROSE, Y., KAWAUCHI, S., OGURA, T. and UMESONO, K. 2000 Aldehyde dehydrogenase 6 , a cytosolic retinaldehyde dehydrogenase prominently expressed in sensory neuroepithelia during development. J.Biol.Chem. 275, 41210-41218.

HALE F. 1933. Pigs born without eye balls. J Hered 24:105-106.

HALILAGIC A, ZILE MH and STUDER M. 2003. A novel role for retinoids in patterning the avian forebrain during presomite stages. Development 130:20392050.

HAMBURGER $V$ and HAMILTON HL. 1951. A series of normal stages in the development of the chick embryo. J Morph 88:49-92.

HASELBECK RJ, HOFFMANN I and DUESTER G. 1999. Distinct functioms for Aldh1 and Raldh2 in the control of ligand production for embryonic retinoid signalling pathways. Dev Genet 25:353-364.

HYATT GA, SCHMITT EA, MARSH-ARMSTRONG N, MCCAFFERY P, DRAGER UC and DOWLING JE. 1996. Retinoic acid establishes ventral retinal characteristics. Development 122:195-204.

HYATT GA, SCHMITT EA, MARSH-ARMSTRONG NRB and DOWLING JE. 1992. Retinoic acid-induced duplication of the zebrafish retina. Proc Natl Acad $\mathrm{SC}$ USA 89:8293-8297.

KASTNER P, GRONDONA JM, MARK M, GANSMULLER A, LEMEUR M, DECIMO D, VONESCH J-L, DOLLE P and CHAMBONP. 1994. Genetic analysis of RXRa developmental function: convergence of RXR and RAR signalling pathways in heart and eye morphogenesis. Cel/78:987-1003.

KASTNER P, MARK M, GHYSELINCK N, KRETZEL W, DUPE V and GRONDONA JM. 1997. Genetic evidence that the retinoid signal is transduced by heterodimeric RXR/RAR functional units during mouse development. Development 124:313326.

KIL SH, STREIT A, BROWN ST, AGRAWAL N, COLLAZO A, ZILE MH and GROVES AK. 2005. Distinct roles for hindbrain and paraxial mesoderm in the induction and patterning of the inner ear revealed by a study of vitamin-Adeficient quail. Dev Bio/285:252-271.

LI H, TIERNEY C, WEN L, WU JY and RAO Y. 1997. A singel morphogenetic field gives rise to two retina primirdia under the influence fo the prochordal plate. Development 124, 603-615.

LOHNES D, MARK M, MENDELSOHN C, DOLLE P, DIERICH A, GORRY P, GANSMULLER A and CHAMBON P. 1994. Function of the retinoic acid receptors (RARs) during development (I) Craniofacial and skeletal abnormalities in RAR double mutants. Development 120:2723-2748.

LUPO G, LIU Y, QIU R, CHANDRARATNA RA, BARSACCHI G, HE RQ and HARRIS WA. 2005. Dorsoventral patterning of the Xenopus eye: a collaboration of Retinoid, Hedgehog and FGF receptor signaling. Development 132:17371748.

MACDONALD R BARTH KA, XU Q, HOLDER N, MIKKOLA I and WILSON SW. 1995. Midline signalling is required for Pax gene regulation and patterning of the eyes. Development 121:3267-3278

MADEN M. 2004. Retinoid signaling during gastrulation. In Gastrulation: from cells to embryo. (Ed. Stern C.D.). New York: Cold Spring Harbor Laboratory Press. p 549-552.

MADEN M, GALE E, KOSTETSKII I and ZILE M. 1996. Vitamin A-deficient quail embryos have half a hindbrain and other neural defects. Curr Bio/6:417-426.
MADEN, M., GRAHAM, A., ZILE, M. and GALE, E. 2000. Abnormalities of somite development in the absence of retinoic acid. Int.J.Dev.Biol. 44, 151-159.

MANNS M, FRITSZCH B. 1991. The eye in the brain: retinoic acid effects morphogenesis of the eye and pathway selection of axons but not the differentiation of the retina in Xenopus laevis. Neurosci Let 127:150-154.

MARSH-ARMSTRONG N, MCCAFFERY P, GILBERT W, DOWLING JE and DRAGER UC. 1994. Retinoic acid is necessary for development of the ventral retina in zebrafish. Proc Natl Acad Sci USA 91:7286-7290.

MATT N, DUPE V, GARNIER JM, DENNEFELD C, CHAMBON P, MARK M and GHYSELINCK NB. 2005. Retinoic acid-dependent eye morphogenesis is orchestrated by neural crest cells. Development 132:4789-4800.

MCCAFFERY P, LEE M-O, WAGNER MA, SLADEK NE and DRAGER U. 1992 Asymmetrical retinoic acid synthesis in the dorsoventral axis of the retina. Development 115:371-382.

MCCAFFERY P, POSCH KC, NAPOLI JL, GUDAS L and DRAGER UC. 1993. Changing patterns of the retinoic acid system in the developing retina. Dev Biol 158:390-399.

MCCAFFERY P, TEMPST P, LARA G and DRAGER UC. 1991. Aldehyde dehydrogenase is a positional marker in the retina. Development 112:693-702.

MCCAFFERY P, WAGNER E, O'NEIL J, PETKOVICH M and DRAGER UC. 1999. Dorsal and ventral territories defined by retinoic acid synthesis, break-down and nuclear receptor expression. Mech Dev 82:119-130.

MEY, J., MCCAFFERY, P. and DRAGER, U. C. 1997. Retinoic acid synthesis in the developing chick retina. J.Neurosci. 17: 7441-7449.

MIC, F. A., MOLOTKOV, A., FAN, X., CUENCA, A. E. and DUESTER, G. 2000 $\mathrm{RALDH} 3$, a retinaldehyde dehydrogenase that generates retinoic acid, is expressed in the ventral retina, otic vesicle and olfactory pit during mouse development. Mech.Dev. 97: 227-230.

MIC FA, MOLOTKOV A, MOLOTKOVA N and DUESTER G. 2004. Raldh2 expression in optic vesicle generates a retinoic acid signal needed for invagination of retina during optic cup formation. Dev Dyn 231: 270-277.

MOLOTKOV A, MOLOTKOVA N and DUESTER G. 2006. Retinoic acid guides eye morphogenetic movements via paracrine signaling but is unnecessary for retinal dorsoventral patterning. Development 133:1901-1910.

NIEDERREITHER K, MCCAFFERY P, DRAGER UC, CHAMBON P and DOLLE P. 1997. Restricted expression and retinoic acid-induced downregulation of the retinaldehyde dehydrogenase type 2 (RALDH-2) gene during mouse development. Mech Dev62:67-78.

QUINLAN R, GALE E, MADEN M and GRAHAM A. 2002. Deficits in the posterior pharyngeal endoderm in the absence of retinoids. Dev Dyn 225:54-60.

REIJNTJES S, BLENTIC A, GALE E and MADEN M. 2005. The control of morphogen signalling: Regulation of the synthesis and catabolism of retinoic acid in the developing embryo. Dev Biol. 285:224-237.

REIJNTJES S, GALE E and MADEN M. 2003. Expression of the retinoic acid catabolising enzyme CYP26B1 in the chick embryo and its regulation by retinoic acid. Gene Expr Patterns 3:621-627.

REIJNTJES S, GALE E and MADEN M. 2004. Generating gradients of retinoic acid in the chick embryo: Cyp26C1 expression and a comparative analysis of the Cyp26 enzymes. Dev Dyn 230:509-517.

RIBES V, WANG Z, DOLLE P and NIEDERREITHER K. 2006. Retinaldehyde dehydrogenase 2 (RALDH2)-mediated retinoic acid synthesis regulates early mouse embryonic forebrain development by controlling FGF and sonic hedgehog signaling. Development 133:351-361.

ROSA FW, WILK AL and KELSEY FO. 1986. Teratogen update: vitamin A congeners. Teratology 33:355-364.

SAKAI Y, LUO T, MCCAFFERY P, HAMADA H and DRAGER UC. 2004. CYP26A1 and CYP26C1 cooperate in degrading retinoic acid within the equatorial retina during later eye development. Dev Bio/276:143-157.

SEN J, HARPAVAT S, PETERS MA and CEPKO CL. 2005. Retinoic acid regulates the expression of dorsoventral topographic guidance molecules in the chick retina. Development 132:5147-5159.

SHENFELT RE. 1972. Morphogenesis of malformations in hamsters caused by retinoic acid: relation to dose and stage at treatment. Teratology 5:103-118.

SHENG HZ and WESTPHAL H 1999. Early steps in pituitary organogenesis. Trends Genet. 15:236-240. 
SIVE HL, DRAPER BW, HARLAND RM and WEINTRUB H. 1990. Identification of a retinoic acid-sensitive period during primary axis formation in Xenopus laevis. Genes Dev 4: 932-942.

SULIK KK, DEHART DB, ROGERS JM and SHERNOFF N. 1995. Teratogenicity of low doses of all-trans retinoic acid in presomite mouse embryos. Teratology51: 398-403.

SUZUKI, S., SHINTANI, T., SAKUTA, H., KATO, A., OHKAWARA, T., OSUMI, N. AND NODA, M. 2000. Identification of RALDH-3, a novel retinaldehyde dehydrogenase, expressed in the ventral region of the retina. Mech Dev. 98: 37-50.

TAKUMA N, SHENG HZ, FURUTA Y, WARD JM, SHARMA K, HOGAN BLM, PFAFF SL, WESTPHAL H, KIMURA S and MAHON KA. 1998. Formation of Rathke's pouch requires dual induction from the diencephalon. Development 125: 4835-4840.

TINI M, OTULAKOWSKI G, BREITMAN ML, TSUI L-C and GIGUERE V. 1993. An everted repeat mediates retinoic acid induction of the $\mathrm{gF}$-crystallin gene: evidence of a direct role for retinoids in lens development. Genes Dev 7: 295307.

TREIER M, GLEIBERMAN AS, O'CONNELL SM, SZETO DP, MCMAHON JA, MCMAHON AP and ROSENFELD MG. 1998. Multistep signalling requirements for pituitary organogenesis in vivo. Genes Dev. 12: 1691-1704.

TREIER M, O'CONNELL S, GLEIBERMAN A, PRICE J, SZETO DP, BURGESS R, CHUANG P-T, MCMAHON AP and ROSENFELD MG. 2001. Hedgehog signalling is required for pituitary gland development. Development 126: 377-386.

WAGNER, E., MCCAFFERY, P. and DRAGER, U. 2000. Retinoic acid in the formation of the dorsoventral retina and its central projections. Dev.Biol. 22
460-470.

WARKANY J, ROTH CB and WILSON JG. 1948. Multiple congenital malformations: a consideration of etiologic factors. Pediatrics 1: 462-471.

WARKANY J SCHRAFFENBERGER E. 1946. Congenital malformations induced in rats by maternal vitamin A deficiency. Arch Ophthalmo/35: 150-169.

WILSON JG, ROTH CB and WARKANY J. 1953. An analysis of the syndrome of malformations induced by maternal vitamin A deficiency. Effects of restoration of vitamin $\mathrm{A}$ at various times during gestation. Am $J$ Anat 92: 189-217.

WILSON L, GALE E, CHAMBERS D and MADEN M. 2004. Retinoic acid and the control of dorsoventral patterning in the avian spinal cord. Dev Bio/269: 433 446.

WILSON L, GALE E and MADEN M. 2003. The role of retinoic acid in the morphogenesis of the neural tube. J Anat203: 357-368.

ZHANG X-M and YANG X-J. 2001. Temporal and spatial effects of sonic hedgehog signalin in chick eye morphogenesis. Dev. Biol. 233: 271-290.

ZILE MH, KOSTETSKII I, YUAN S, KOSTETSKAIA E, ST.AMAND, TR, CHEN, Y$P$ and JIANG, W. 2000. Retinoid signaling is required to complete the vertebrate cardiac left/right asymmetry pathway. Dev.Biol. 223: 323-338.

\section{Related Int. J. Dev. Biol. articles}

See our recent Special Issue on Eye Development edited by J. Piatigorski and R. Grainger at: http://www.ijdb.ehu.es/web/contents. php?vol=48\&issue=8-9

TBX1, a DiGeorge syndrome candidate gene, is inhibited by retinoic acid. Lifeng Zhang, Tao Zhong, Yuexiang Wang, Qiu Jiang, Houyan Song and Yonghao Gui. Int. J. Dev. Biol. (2006) 50: 55-61

The role of XTRAP-gamma in Xenopus pronephros development. Dong-hui Li, Techuan Chan, Reiko Satow, Shinji Komazaki, Kouhei Hashizume and Makoto Asashima. Int. J. Dev. Biol. (2005) 49: 401-408

Antero-posterior patterning of the vertebrate digestive tract: $\mathbf{4 0}$ years after Nicole Le Douarin's PhD thesis. Anne Grapin-Botton. Int. J. Dev. Biol. (2005) 49: 335-347

Regulation of gene expression by Pax6 in ocular cells: a case of tissue-preferred expression of crystallins in lens. Ales Cvekl, Ying Yang, Bharesh K. Chauhan and Kveta Cveklova. Int. J. Dev. Biol. (2004) 48: 829-844

Retinoic acid repatterns axolotl lateral line receptors. Melissa $A$ Gibbs and $R$ Glenn Northcutt. Int. J. Dev. Biol. (2004) 48: 63-66

Exogenous retinoic acid induces a stage-specific, transient and progressive extension of Sonic hedgehog expression across the pectoral fin bud of zebrafish. Lisa Hoffman, Jennifer Miles, Fabien Avaron, Lynda Laforest and Marie-Andrée Akimenko. Int. J. Dev. Biol. (2002) 46: 949-956

Retinoic acid and limb regeneration - a personal view. Malcolm Maden. Int. J. Dev. Biol. (2002) 46: 883886.

Retinoic acid response element in HOXA-7 regulatory region affects the rate, not the formation of anterior boundary expression. Myoung H Kim, Jae S Shin, Sungdo Park, Man-Wook Hur, Mi-Ok Lee, Hyoungwoo Park and Chul-Sang Lee

Int. J. Dev. Biol. (2002) 46: 325-328

Expression pattern of PAX3 and PAX6 genes during human embryogenesis. J Terzic and M SaragaBabic. Int. J. Dev. Biol. (1999) 43: 501-508 